\title{
Correction to: Novel SNP markers in InvGE and Sssl genes are associated with natural variation of sugar contents and frying color in Solanum tuberosum Group Phureja
}

\author{
D. Duarte-Delgado ${ }^{1,2}$, D. Juyó ${ }^{1}$, C. Gebhardt ${ }^{3}$, F. Sarmiento ${ }^{4}$ and T. Mosquera-Vásquez ${ }^{5^{*}}$
}

\section{Correction}

After the publication of this work [1], we detected some mistakes in the reverse primers of InvGE and $B M Y-8 / 2$ and in the forward primer of PWD shown in Table 1. Additionally, we made some editions in the primer sequences from Additional files 2 and 3. Please see the corrected Table 1 and Additional files 2 and 3 below.

\section{Additional files}

Additional file 2: Amplicon sequences of candidate genes with associations to sugar contents and frying color in Solanum tuberosum Group Phureja with the SNP alleles and SNP positions in the potato reference genome (version 4.03) $[28,43]$. The sequences were retrieved from the SPUD data base [44]. Primer sequences are underlined, markers with associations are highlighted in blue and SNPs previously reported for tetraploid potatoes are shown in italics. The numbers indicate the position of the sequence and the SNPs in the chromosomes (chr). Exonic regions are represented with red letters while introns are represented in black letters according to the gene models from the SPUD data base [44]. (DOCX $23 \mathrm{~kb}$ )

Additional file 3: Amplicon sequences of additional candidate genes studied in Solanum tuberosum Group Phureja with the SNP alleles and SNP positions in the potato reference genome (version 4.03) [28, 43]. The sequences were retrieved from the SPUD data base [44]. Primer sequences are underlined and the SNPs previously reported for tetraploid potatoes are highlighted in yellow. Exonic regions are represented with red letters while introns are represented in black letters according to the gene models from the SPUD data base [44]. (DOCX $26 \mathrm{~kb})$

\section{Author details}

${ }^{1}$ Faculty of Agricultural Sciences, Agronomy Department, National University of Colombia, Bogotá, Colombia. '2Present address: INRES-Plant Breeding, University of Bonn, Bonn, Germany. ${ }^{3}$ Department of Plant Breeding and Genetics, Max Planck Institute for Plant Breeding Research, Cologne, Germany. ${ }^{4}$ Faculty of Sciences, Biology Department, National University of Colombia, Bogotá, Colombia. ${ }^{5}$ Faculty of Agricultural Sciences, Agronomy Department, National University of Colombia, Bogotá, Colombia.

Received: 26 September 2017 Accepted: 4 October 2017

Published online: 20 October 2017

\section{Reference}

1. Duarte-Delgado D, Juyó D, Gebhardt C, Sarmiento F, Mosquera-Vásquez T. Novel SNP markers in InvGE and Sss/ genes are associated with natural variation of sugar contents and frying color in Solanum tuberosum group Phureja. BMC Genomics. 2017;18:23.

\footnotetext{
* Correspondence: tmosquerav@unal.edu.co

${ }^{5}$ Faculty of Agricultural Sciences, Agronomy Department, National University of Colombia, Bogotá, Colombia
} 
Table 1 Loci (PGSC0003DMG********) from the reference genome [28, 43] analyzed in 112 Solanum tuberosum Group Phureja accessions

\begin{tabular}{|c|c|c|c|c|c|c|}
\hline $\begin{array}{l}\text { Gene Acronym } \\
\text { (GenBank Accesion No.) }\end{array}$ & Locus & Chr. & Primer Sequences $5^{\prime}-3^{\prime}$ & $\mathrm{Ta}\left({ }^{\circ} \mathrm{C}\right)$ & $\begin{array}{l}\text { Amplicon } \\
\text { size (bp) }\end{array}$ & $\begin{array}{l}\text { No. SNPs Scored } \\
\text { (Scored previously) }^{e}\end{array}$ \\
\hline $\begin{array}{l}\text { Stp23 }(\mathrm{PHO} 1)^{a} \\
(\mathrm{D} 00520)\end{array}$ & PGSC0003DMG400007782 & III & $\begin{array}{l}{ }^{*} \text { f-cagatatgttacatactctacc } \\
\text { r-tcattagtcacaactttatcgg }\end{array}$ & 59 & 963 & $4(0)$ \\
\hline $\begin{array}{l}\text { StpL }(\mathrm{PHO} 1)^{\mathrm{a}} \\
(\mathrm{X} 73684)\end{array}$ & PGSC0003DMG400028382 & v & $\begin{array}{l}{ }^{*} \text { f-ttacattgcacaagcacaagc } \\
\text { r-gtgtacatacaatactctatcc }\end{array}$ & 57 & 982 & $14(1)[67]$ \\
\hline SSSI (Y10416) & PGSC0003DMG402018552 & III & $\begin{array}{l}\text { f-aacaataggaatttaccataacc } \\
{ }^{*} \text {-atattcccaaacaaaacagagc }\end{array}$ & 57 & 970 & $12(0)$ \\
\hline $\begin{array}{l}\operatorname{lnvGE}(\text { INV-CW })^{a} \\
(\text { AJ133765) }\end{array}$ & PGSC0003DMG400008943 & IX & $\begin{array}{l}\text { f-caattcttcgattcttcatagg } \\
{ }^{*} \text { r-acagcacctatgtattataatgg }\end{array}$ & 57 & 798 & 9 (8) [39] \\
\hline \multirow[t]{3}{*}{$\begin{array}{l}\text { Pain 1 (INV) } \\
(X 70368)\end{array}$} & PGSC0003DMG400013856 & III & $\begin{array}{l}\text { f-catacattacactatagatcc } \\
{ }^{*} \text {-aattgaagcagatcatgtagg }\end{array}$ & 56 & 926 & $5(2)[39,40]$ \\
\hline & & & f-caaaatgaatacatattaagagg ${ }^{b}$ & 56 & 707 & $4(0)$ \\
\hline & & & ${ }^{*}$ r-cttaagcagttgcttagagc & & & \\
\hline \multirow[t]{4}{*}{ UGPase (D00667) } & PGSC0003DMG401013333 & $X I$ & f-atgatgttctccacttaaaagc & 56 & 805 & $11(1)[42]$ \\
\hline & & & ${ }^{*_{r} \text {-tttcagattttcagaagagagg }}$ & & & \\
\hline & & & ${ }^{*}$-tgattaacgatactatacgtcc & 56 & 931 & $12(1)[42]$ \\
\hline & & & r-ttaaaacttccttatactatatgg & & & \\
\hline \multirow[t]{2}{*}{ GWD (Y09533) } & PGSC0003DMG400007677 & V & f-ttctgttatctacttagttacg & 56 & 992 & $5(3)[7,41]$ \\
\hline & & & ${ }^{*}$ r-gttttatatcttgcttctttgg & & & \\
\hline \multirow[t]{2}{*}{ BMY-8/2 (AF393847) } & PGSC0003DMG400001855 & VIII & f-gctactggagcatggtgacaga ${ }^{c}$ & 57 & 560 & $9(9)[5]$ \\
\hline & & & ${ }^{*}$ r-ttacatagaggtctgtcctgcttgag & & & \\
\hline \multirow[t]{2}{*}{ PWD (AY747068) } & PGSC0003DMG400016613 & IX & ${ }^{*}$-ggtctgatgatctatctgattg $c^{c}$ & 57 & 871 & $16(16)[7,41]$ \\
\hline & & & r-cgacatcttgaggagaaccaaactt & & & \\
\hline \multirow[t]{2}{*}{$\operatorname{LapN}(X 77015)$} & PGSC0003DMG400007831 & $X \|$ & f-gcttcctggtcttggctc ${ }^{d}$ & 60 & 985 & 8 (3) [37] \\
\hline & & & ${ }^{*}$ r-gataggcatacgcagccaggtcagaaatcaa & & & \\
\hline
\end{tabular}

Chr, chromosome; Ta, annealing temperature

* Primer used for amplicon sequencing

a These parenthesis include the alternative acronyms used in the starch-sugar interconversion pathway scheme adapted by Schreiber et al. [7]

${ }^{b}$ From a region downstream of the Pain 1 candidate gene

c From Schreiber et al. [7]

${ }^{d}$ From Fischer et al. [37]

e The studies where the SNPs or the related amino acid changes were previously reported in tetraploid potatoes are indicated 